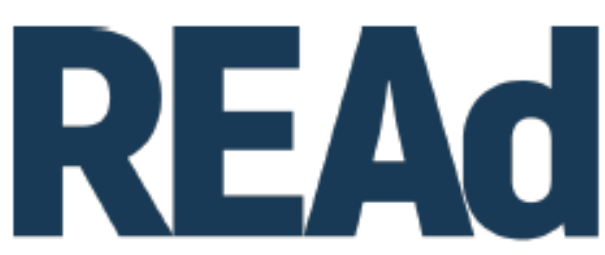

Revista Eletrônica de Administração

\title{
A RELAÇÃO ENTRE INVESTIMENTO EM PROPAGANDA E O VALOR DA FIRMA: UMA REVISÃO SISTEMÁTICA E UMA AGENDA DE PESQUISA PARA FUTUROS ESTUDOS ${ }^{1}$
}

\author{
Ricardo Saraiva Frio ${ }^{2}$ \\ Thiago dos Santos Glasenapp ${ }^{3}$ \\ Rodrigo Heldt Silveira ${ }^{4}$ \\ Fernando Bins Luce Lu $^{5}$
}

http://dx.doi.org/10.1590/1413-2311.272.96038

\begin{abstract}
RESUMO
Um desafio aos profissionais e pesquisadores de marketing é demonstrar o impacto de suas ações no valor das empresas. $\mathrm{O}$ alto investimento das organizações em propaganda gera necessidade de demonstrar os benefícios financeiros dos esforços e investimentos neste instrumento de marketing. Diante do elevado número de artigos discutindo esse tópico, uma revisão sistemática acerca da relação entre investimento em propaganda e o valor da firma foi conduzida com os objetivos de avaliar os resultados obtidos em diferentes categorias temáticas acerca do tópico e avaliar o efeito dos investimentos em propaganda sobre diferentes métricas de valor da firma. Utilizando as bases de dados EBSCO, Google Acadêmico e Web of Science foram buscados artigos publicados entre 1997 e 2019, atingindo, assim, uma base final de 43

\footnotetext{
${ }^{1}$ Recebido em 30/8/2019, aprovado em 29/10/2019.

${ }^{2}$ Universidade Federal do Rio Grande - Instituto de Ciências Econômicas, Administrativas e Contábeis; Rio Grande - RS (Brasil); http://orcid.org/0000-0003-4536-4687; ricardo.frio@ yahoo.com.br

${ }^{3}$ Universidade Federal do Rio Grande do Sul (UFRGS) - Programa de Pós-Graduação em Administração; Porto Alegre - RS (Brasil); http://orcid.org/0000-0003-4713-3700; thiago.glasenapp@ gmail.com

${ }^{4}$ Universidade Federal do Rio Grande do Sul (UFRGS) - Programa de Pós-Graduação em Administração; Uniritter; Porto Alegre - RS (Brasil); http://orcid.org/0000-0002-3435-8962; rodrigoheldt@ gmail.com

${ }^{5}$ Universidade Federal do Rio Grande do Sul - Programa de Pós-Graduação em Administração - Porto Alegre RS (Brasil); http://orcid.org/0000-0003-2589-2845; fernando.luce@ufrgs.br
} 
artigos para avaliação. Os resultados evidenciam majoritariamente efeitos positivos da comunicação no valor da firma, embora alguns resultados contraditórios também tenham sido encontrados. Variáveis com potencial para afetar essa relação também foram observadas, tais como cultura, setor e tamanho da empresa. Adicionalmente, foi possível identificar uma escassez de estudos brasileiros sobre o tema. Por fim, propôs-se uma agenda para pesquisas futuras a fim de se obter maiores avanços nos estudos acerca do tema.

Palavras-chave: Investimento em propaganda. Valor da firma. Métrica de marketing. Rrevisão sistemática. Produtividade de marketing.

\title{
THE RELATIONSHIP BETWEEN ADVERTISING SPENDING AND THE VALUE OF THE FIRM: A SYSTEMATIC REVIEW AND A RESEARCH AGENDA FOR FUTURE STUDIES
}

\begin{abstract}
One of the challenges for marketers is to demonstrate the impact of their activities on firm value. The high investment of organizations in advertising generates a need for marketers to demonstrate the financial outcomes of efforts and investments in this marketing tool. Given the large number of articles discussing this topic, a systematic review about the relationship between advertising investment and firm value was conducted with the objective of evaluating the results obtained in different thematic categories on the topic and evaluating the effect of advertising investments on different firm value metrics. Using EBSCO, Google Scholar and Web of Science databases, we searched articles published between 1997 and 2019, thus reaching a final base of 43 articles for evaluation. The results mostly show positive effects of communication on firm value, although some contradictory results were also found. Variables with potential to affect this relationship were also observed, such as culture, type of industry and company size. Additionally, it was possible to identify a shortage of Brazilian studies on the subject. Finally, an agenda for future research was proposed in order to obtain further advances in the comprehension of the subject.
\end{abstract}

Keyword: Advertising spending. Firm value. Marketing metrics. Systematic review. Marketing productivity. 


\section{LA RELACIÓN ENTRE LA INVERSIÓN DE PROPAGANDA Y EL VALOR DE LA EMPRESA: UNA REVISIÓN SISTEMÁTICA Y UNA AGENDA DE INVESTIGACIÓN PARA ESTUDIOS FUTUROS}

\section{RESUMEN}

Un desafío para los especialistas en marketing e investigadores es demostrar el impacto de sus acciones en el valor de las empresas. La alta inversión de las organizaciones en publicidad genera la necesidad de demostrar los beneficios financieros de los esfuerzos e inversiones en esta herramienta de marketing. Dado el gran número de artículos que discuten este tema, se realizó una revisión sistemática sobre la relación entre la inversión publicitaria y el valor de la empresa con el objetivo de evaluar los resultados obtenidos en diferentes categorías temáticas sobre el tema y evaluar el efecto de las inversiones publicitarias sobre diferentes métricas de valor de la empresa. Utilizando las bases de datos EBSCO, Google Scholar y Web of Science se buscaron artículos publicados entre 1997 y 2019, alcanzando así una base final de 43 artículos para evaluación. Los resultados muestran principalmente efectos positivos de la comunicación en el valor de la empresa, aunque también se encontraron algunos resultados contradictorios. También se observaron variables con potencial para afectar esta relación, como la cultura, el sector y el tamaño de la empresa. Además, fue posible identificar una escasez de estudios brasileños sobre el tema. Finalmente, se propuso una agenda para futuras investigaciones con el fin de obtener más avances en los estudios sobre el tema.

Palabras clave: Inversión publicitaria. Valor de la empresa. Métricas de marketing. Revisión sistemática. Productividad de marketing.

\section{INTRODUÇÃO}

Profissionais e acadêmicos da área de marketing, cada vez mais, são impelidos a demonstrarem de maneira prática os impactos das ações de marketing no resultado das empresas (REIBSTEIN et al. 2009; STEWART, 2009). Para Katsikeas et al. (2016), uma questão central ao marketing é o seu papel em explicar a performance da empresa. Nessa linha, Srinivasan e Hanssens (2009) acreditam que os profissionais e pesquisadores da área estão 
diante de um maior clamor para comunicar o valor criado aos acionistas a partir de suas ações e políticas. Desse modo, nota-se uma aproximação da pesquisa em marketing com a pesquisa em finanças (HYMAN e MATHUR, 2005), a partir da perspectiva proposta por Hanssens, Rust e Srivastava (2009), de que as atividades exercidas pelo marketing devem contribuir para o aumento do valor da firma.

Propaganda e comunicação são áreas do marketing que constantemente recebem elevados investimentos dentro das organizações, necessitando assim demonstrar sua contribuição para maior performance da empresa (FRENNEA, HAN e MITTAL, 2019; SHIMP e ANDREWS, 2012). Ainda que empresas que invistam mais em propaganda apresentem melhor performance ${ }^{6}$ (JOHNSON e SOENES, 2003), pesquisas começam a apontar para um efeito teto onde o investimento além do necessário em propaganda, pode gerar ineficiência por parte das empresas (CHEONG, GREGORIO e KIM, 2014). Ainda de acordo com os autores, este fenômeno de ineficiência no investimento em propaganda está evoluindo ao longo dos anos.

A partir disso, o estudo de propaganda e seus efeitos tem recebido maior atenção na academia. Em editorial no International Journal of Advertsing: The Review of Marketing Communications, Taylor (2010) enfatiza a necessidade de pesquisas que tratem sobre o retorno de investimento em programas de comunicação integrada de marketing. Mais recentemente, em 2017, o European Journal of Marketing dedicou uma edição inteira ao debate da comunicação integrada de marketing. Kitchen (2017, pg. 394) aponta para uma evolução da comunicação integrada de marketing e que "medir o retorno do investimento se tornou mais direto, a partir dos telefones celulares, e mais complexos, já que muitos outros canais de mídia se proliferam". O estudo do impacto da propaganda nos resultados empresariais é, portanto, de extrema importância prática e de notável interesse acadêmico.

Apesar da relevância do tema e do crescimento do interesse no tópico, é importante observar que a relação entre investimento em propaganda e o valor da firma, apresenta algumas lacunas. De acordo com Shah e Akbar (2008), os estudos relacionados à investimento em propaganda e o valor da firma apresentam resultados divergentes. De fato, enquanto alguns autores apresentam resultados positivos na relação entre o investimento em propaganda e o valor da firma (e.g. JOSHI e HANSSENS, 2010), outros apontam que o aumento de investimentos em determinadas ferramentas de comunicação leva ao declínio de eficácia de

\footnotetext{
${ }^{6}$ Considerando as medidas de desempenho Sharpe Ratio, Jensen's alpha e Valor Econômico Adicionado.
} 
outras (e.g. SRIDHAR et al. 2016). Há também casos onde a relação entre investimento em propaganda e o valor da firma não é observada (TULI, MUKHERJEE e DEKIMPE, $2012^{7}$ ).

A partir disso, se faz relevante um melhor e mais aprofundado entendimento sobre a relação entre o investimento em comunicação de marketing e o valor da firma. Assim, uma revisão sistemática acerca da relação entre investimento em propaganda e o valor da firma foi conduzida, afim de congregar os esforços acadêmicos feitos sobre o tópico e sedimentar as posições proeminentes entre os autores da área.

Este artigo contribui de maneira significativa para a literatura existente. Primeiro, através da utilização de uma revisão sistemática baseada no trabalho de Wolfswinkel, Furtmueller e Wilderom (2011), foi possível avaliar os resultados da propaganda obtidos em diferentes categorias temáticas (i.e. investimento em propaganda, competição). Segundo, avalia o efeito dos investimentos em propaganda sob diferentes métricas de valor da firma (i.e. Q de Tobin, retorno de ações). Terceiro, atualiza e dá continuidade aos esforços de Shah e Akbar (2008). Até o momento, pesquisas que avaliam retorno de investimento em propaganda no valor da firma limitaram-se ao período de $1964-2004^{8}$. Ao considerar um período mais recente da publicação dos artigos (de 1997 a 2019), este artigo avalia também a comunicação moderna, impactada e alterada devido aos avanços tecnológicos dos últimos 15 anos. Todos estes esforços vão ao encontro de Palmatier, Houston e Hulland (2018), que acreditam que artigos de revisão podem ser úteis ao progresso científico do marketing. Por fim, este artigo oferece uma quarta e última contribuição, ao propor uma agenda de pesquisa orientando esforços de pesquisadores da área sobre futuros caminhos a serem seguidos.

\section{REFERENCIAL TEÓRICO}

\subsection{INVESTIMENTO EM PROPAGANDA}

O investimento em propaganda no marketing pode ser executado através de diversas ferramentas (e.g. televisão, rádio, patrocínio, entre outras), cada qual destinada a um objetivo específico e com capacidade de alcançar públicos distintos (SHIMP e ANDREWS, 2013). Cabe notar, no entanto, que distintas mídias podem afetar umas às outras, conforme identificam Sridhar et al. (2016). Individualmente os três tipos de mídias por eles utilizados (online,

\footnotetext{
${ }^{7}$ Frente a um crescimento inesperado.

${ }^{8}$ Os autores não explicitam no texto o período de análise. Considerou-se 1964-2004 com base nos resultados apresentados.
} 
nacional e regional) afetam positivamente no valor da firma, contudo, o aumento do efeito de uma acaba por canibalizar a eficácia das outras. Assim, os gestores devem identificar um nível ótimo de investimento para evitar gastos elevados e ineficazes (CHEONG, GREGORIO e KIM, 2014).

O valor financeiro das empresas pode ser afetado por um grande número de eventos sejam eles feitos deliberadamente pela empresa ou condições de mercado alheias às ações da empresa - que geram expectativas (positivas ou negativas) aos investidores com potenciais ganhos futuros -, pois o investimento em propaganda é uma ação empresarial que gera expectativa aos investidores, influenciando seu comportamento (JOSHI e HANSSENS, 2010). A partir de resultados anteriores, Sridhar et al. (2016) explicam que o dispêndio em propaganda pode aumentar as vendas, melhorando assim a performance das empresas.

Liu, Shankar e Yun (2017), identificam que um maior investimento em propaganda de marca, após uma ação de recall, reduz os efeitos negativos do recall e do retorno anormal de ações. Os autores argumentam que o investimento em propaganda de marca gera efeitos positivos tanto nos consumidores quanto nos investidores. Ainda argumentam que uma ênfase na comunicação demonstra compromisso em aumentar o valor da marca e os investidores tornam-se menos propensos a esperar um impacto negativo no fluxo de caixa. O maior brand equity, métrica sobre valor da marca, afeta positivamente a performance da firma (PETERSON e JEONG, 2010).

De modo geral, a literatura demonstra a importância do investimento em propaganda, devido aos efeitos positivos sobre vendas, valor da marca e do efeito positivo dessa ferramenta gerado sobre acionistas. No entanto, é necessário que esse investimento seja feito de maneira criteriosa, a fim de manter a eficiência das comunicações e evitar gastos desnecessários.

\subsection{MÉTRICAS DE MARKETING}

Métrica pode ser entendida como "um sistema de medição que quantifica uma tendência, dinâmica ou caraterística [...] as métricas incentivam o rigor e a objetividade. Elas possibilitam comparar observações entre regiões e períodos de tempo" (BENDLE et al. 2015, pg. 13).

A partir da década de 1990, pesquisadores passaram a apontar para a necessidade de a área de marketing ser responsável na mensuração dos resultados de suas atividades (GÖK e HACIOGLU, 2010; HOMBURG et al. 2015; RUST, LEMON e ZEITHAML, 2004; SHETH e 
SISODIA, 1995a, 1995b; SRIVASTAVA, SHERVANI e FAHEY, 1998). Sheth e Sisodia (1995a, 1995b), trouxeram essa necessidade para o centro das atenções, afirmando que as ações de marketing não eram adequadamente mensuradas e que a área carecia de métricas confiáveis.

Tal constatação foi seguida por grandes esforços da área, nas duas décadas seguintes, no desenvolvimento de métricas de marketing e na busca por melhoria nos níveis de eficiência da área. Autores passaram a propor modelos de mensuração de produtividade em marketing (RUST et al. 2004; SRIVASTAVA, SHERVANI e FAHEV, 1998) e a continuidade no desenvolvimento da área na capacidade de mensuração de performance ainda é reiterada em artigos mais recentes (GÖK e HACIOGLU, 2010; HOMBURG et al. 2015).

Esse anseio acadêmico na criação de métricas que auxiliassem na avaliação do resultado dos esforços da área ocorre concomitantemente ao aumento da necessidade de que profissionais de marketing quantifiquem o valor de seus produtos, consumidores e canais de distribuição. Isso ocorre, pois esse profissional é cada vez mais responsável pelos resultados financeiros de suas decisões (BENLER et al. 2016).

O desempenho empresarial é avaliado através de métricas que quantificam os resultados da empresa. Contudo, não existe uma única métrica. Uma perspectiva dominante aos acadêmicos de marketing utiliza abordagens baseadas em vendas em função da maior disponibilidade de dados. No entanto, outras abordagens consideram lucros e valor de marca (KNOWLES e AMBLER, 2010). Na empresa, a escolha da métrica mais adequada ocorre com base em critérios próprios (AMBLER, KOKKINAKI e PUNTONI, 2004).

Uma métrica comumente citada é o brand equity, ou o valor da marca. A marca compreende o nome, símbolo, logo e imagem que identifica um bem na mente do cliente (BENDLE et al. 2015). A mensuração do valor da marca, no entanto, ainda é tema de debate na academia e muitos métodos já foram propostos. (DATTA, AILAWADI e VAN HEERDE, 2017; OLIVEIRA, SILVEIRA e LUCE, 2015,). Embora classificações alternativas para os modelos de valor da marca existam na literatura de marketing, Christodoulides e De Chernatony (2010) identificam duas grandes vertentes para a mensuração do valor da marca: (1) Valor da marca baseado no consumidor, que avalia as marcas a partir de dimensões que representem as percepções e preferências do consumidor e (2) Valor da marca baseado na firma, que avalia as marcas a partir da mensuração do valor monetário relacionado a mesma.

\subsection{VALOR DA FIRMA}


O grande número de artigos publicados desde a década de 1980 (KATSIKEAS et al. 2016) que abordam os impactos da atividade de marketing na performance empresarial evidencia esforços da área de marketing na pesquisa sobre o tema. Morgan (2012), reforça que os profissionais de marketing devem convergir esforços na busca de maior performance empresarial - seja ela de mercado ou financeira.

Contudo, performance empresarial é um conceito bastante amplo, abarcando uma série de métricas como satisfação, sucesso de novos produtos e lucro (KATSIKEAS et al. 2016). O valor da firma, enquanto uma medida de performance, pode ser associado com medidas que consideram a capitalização de mercado e retorno de ações. (EDELING e FISCHER, 2016). O valor da firma também pode ser considerado o resultado final do desempenho da empresa que irá determinar os ganhos (ou expectativas de ganhos), por parte dos investidores, gerando maior valor para companhia (KATSIKEAS et al. 2016).

Dessa forma, valor da firma pode ser entendido como um termo genérico que considera a performance empresarial a partir da valoração da empresa no mercado de ações, podendo ser mensurado a partir de métricas como retorno de ações e Q de Tobin, bem como a partir de uma análise longitudinal ou de eventos (KATSIKEAS et al. 2016; SRINIVASAN e HANSSENS, 2009).

A partir da teoria de ativos baseados em mercado, observa-se que a empresa que apresenta maior investimento em propaganda, tende a aumentar sua performance de mercado ao criar uma barreira aos concorrentes (KIM, KIM e O’NEILL, 2013). Além disso, empresas que investem mais em publicidade sinalizam ao mercado compromisso com maior fluxo de caixa, e, assim, ganhos futuros aos investidores (JOSHI e HANSSENS, 2010).

\section{MÉTODO DE PESQUISA}

Buscando-se sedimentar a compreensão atual da área de marketing acerca da relação entre investimento em propaganda e o valor da firma, optou-se por realizar uma revisão sistemática da literatura vigente sobre o tópico. Palmatier, Houston e Hulland (2018) acreditam que artigos de revisão são úteis ao progresso científico em marketing.

Para a execução desta revisão sistemática, adotaram-se os passos sugeridos por Wolfswinkel, Furtmueller e Wilderom (2011) e a aplicação prática desse método feita por Broilo e Espartel (2016). Assim, foram realizados os seguintes passos: (1) definição das bases de dados a serem utilizadas; (2) definição dos critérios para busca e seleção dos trabalhos; (3) 
refinamento do conjunto de estudos selecionados; (4) Análise por meio de codificação dos dados captados; e (5) estruturação dos achados. As sessões a seguir abordam mais profundamente cada uma destas etapas.

\subsection{BASES DE DADOS E CRITÉRIOS DE BUSCA}

Para a busca inicial dos estudos, foram utilizadas as bases EBSCO, Web of Science e Google Acadêmico. Como período de busca, foram considerados válidos os papers relacionados ao tema escritos entre 1997 e $2019^{9}$. Observa-se que essas bases compreendem um volume aceitável de dados e são utilizadas em estudos de gestão e de marketing que realizam revisões e compilações da literatura (e.g., BRINCKMANN, GRICHNIK e KAPSA, 2010; CHANG e TAYLOR, 2016; FETSCHERIN e HEINRICH, 2015).

Os filtros de busca foram definidos através da identificação de termos-chave em estudos anteriores (e.g. JOSHI e HANSSENS, 2010; SRIDHAR et al. 2016). Os termos utilizados foram "advertising" e "firm value" e foram considerados os artigos nos quais esses termos aparecessem em seu título, resumo e palavras-chave. Como resultado final foram obtidos 165 artigos.

\subsection{REFINAMENTO DE SELEÇÃO E ANÁLISE}

Após o filtro inicial, foram excluídos os artigos não acadêmicos ou incompletos, (resumos ou intenção de pesquisa) e também artigos em duplicidade entre bases, totalizando 52 artigos. Um total de 30 working papers não puderam ser acessados. Os critérios para a busca obtiveram 22 artigos duplicados, ou seja, apareceram mais de uma vez nos resultados ou o mesmo artigo foi publicado em um congresso e em uma revista. Nessa situação, optou-se por analisar o artigo da revista. Por fim, 18 artigos foram retirados por não apresentarem relação com o tema. No total, 43 artigos permaneceram para análise. Um resumo do processo de seleção e análise dos trabalhos, é apresentada na Tabela 1.

\section{Quadro 1 - Resumo dos artigos encontrados}

\footnotetext{
${ }^{9}$ As buscas ocorreram até março de 2019.
} 


\begin{tabular}{|c|c|c|c|c|c|c|c|}
\hline & $\begin{array}{l}\text { Número } \\
\text { inicial } \\
\text { de } \\
\text { artigos }\end{array}$ & $\begin{array}{l}\text { Artigos } \\
\text { sem } \\
\text { relação } \\
\text { com o } \\
\text { tema }\end{array}$ & $\begin{array}{l}\text { Artigos não } \\
\text { acadêmicos } \\
\text { ou } \\
\text { incompletos }\end{array}$ & $\begin{array}{l}\text { Artigos } \\
\text { em } \\
\text { outra } \\
\text { base }\end{array}$ & $\begin{array}{l}\text { Working } \\
\text { papers } \\
\text { sem } \\
\text { acesso }\end{array}$ & $\begin{array}{c}\text { Artigos } \\
\text { duplicados }\end{array}$ & $\begin{array}{c}\text { Número } \\
\text { final de } \\
\text { artigos } \\
\text { analisados }\end{array}$ \\
\hline EBESCO & 48 & 6 & 4 & 0 & 1 & 18 & 19 \\
\hline $\begin{array}{l}\text { Web of } \\
\text { Science }\end{array}$ & 30 & 3 & 0 & 17 & 0 & 0 & 10 \\
\hline $\begin{array}{c}\text { Google } \\
\text { Acadêmico }\end{array}$ & 87 & 9 & 2 & 29 & $29^{10}$ & 4 & 14 \\
\hline Total & 165 & 18 & 6 & 46 & 30 & 22 & 43 \\
\hline
\end{tabular}

A partir da análise dos artigos, foram definidas as categorias temáticas abordadas: (1) investimento em propaganda; (2) competição; (3) sinergia/canibalização; (4) propaganda enganosa; e (5) gestão de crise.

Artigos que debatem performance, métricas financeiras e valor da firma (KATSIKEAS et al. 2016; OLIVEIRA et al. 2010; SRINIVASAN e HANSSENS, 2009) foram utilizados para caracterizar o tipo de dado utilizado na análise (longitudinal ou estudo de evento) e métricas usadas para determinar o valor da firma. O resultado dessa análise é explorado no próximo capítulo.

\section{RESULTADOS}

A análises dos resultados é realizada em três etapas. Primeiramente, é conduzida uma análise descritiva dos estudos analisados com o intuito de caracterização da amostra. Após, acordo com as categorias temáticas identificadas na revisão, os principais resultados identificados em cada categoria temática são apresentados. Por fim, os resultados acerca dos efeitos de investimentos em propaganda na performance das empresas são analisados de acordo com a métrica de performance da firma utilizada.

\subsection{CARACTERIZAÇÃO DA AMOSTRA}

A verificação das fontes de publicação dos periódicos ocorreu a partir de uma análise de frequência gerada a partir do software JASP®. De acordo com os resultados, as principais

\footnotetext{
${ }^{10}$ Devido aos critérios de busca da ferramenta, não foi possível identificar nesses trabalhos se as palavras de busca encontravam-se no título ou no resumo ou nas palavras-chave.
} 
fontes de publicação são: Journal of Marketing (8 artigos), Journal of Academy of Marketing Science, Journal of Marketing Research e Management Science (3 artigos). Ao separar as fontes (periódicos e congressos) por área, identificou-se uma maior parte de artigos publicados em fontes focadas em marketing (21), Gestão (7), contabilidade finanças (5), comunicação (3), turismo (2) e demais áreas ( 1 cada) (e.g. economia, engenharia e internet).

Observa-se que apesar do chamamento de Taylor (2010), para artigos que testem o retorno do investimento em publicidade, a temática aparenta um grande foco e escopo aos periódicos de marketing. Observa-se ainda que o elevado número de artigos publicados no Journal of Marketing está relacionado com a preocupação do periódico com a análise de "problemas reais" das empresas e suas práticas, foco reiterado em recente editorial (MOORMAN et al. 2019).

Com relação ao ano de publicação, foram identificados os seguintes resultados: sete em 2018; seis artigos publicados em 2016; cinco em 2011 e 2014; quatro em 2010; três em 2009 e 2012; dois em 2017; e um cada um dos demais anos identificados (1997, 2001, 2003, 2005, 2006, 2013, 2015 e 2019).

A análise do período de publicação, torna evidente um aumento no número de artigos relacionados ao tema nos últimos anos. Isso pode ser explicado pelo chamado de alguns autores e pela necessidade do marketing em justificar os elevados investimentos na área (KUMAR, 2015; RUST et al. 2004), além da aproximação entre teoria e prática (KUMAR, 2017; MOORMAN et al. 2019). Em outras palavras, os pesquisadores identificam a necessidade de pesquisas que justifiquem os investimentos em propaganda, atendendo a uma necessidade da academia e do mercado.

Além de considerar o investimento em propaganda, três artigos investigaram competição, dois investigaram sinergia/canibalização e as outras categorias de estudo apresentaram um artigo (propaganda enganosa e gestão de risco). Notou-se também que, em alguns artigos, mais de um tópico é debatido. A vasta maioria dos trabalhos (35 artigos) tratam a propaganda de forma genérica, sem especificar ferramentas. A metodologia preferencial dos estudos foi a análise de dados longitudinais (38 artigos), enquanto estudo de eventos foi utilizado em 7 pesquisas. Observa-se ainda que dois estudos utilizaram as duas metodologias.

Ao considerar as métricas na presente análise, buscou-se, em um primeiro momento, identificar o que os autores do artigo denominam valor da firma, bem como sua relação com investimentos em propaganda. Contudo, como forma de ampliar o conhecimento e os achados, computou-se também estudos que tenham demonstrado o impacto do investimento em 
propaganda em outras métricas de performance da firma estipuladas por Katsikeas et al. (2016); Oliveira et al. (2010); e Srinivasan e Hanssens (2009) - mesmo que não relacionadas diretamente ao valor da firma. Isso permite maior entendimento sobre quais métricas são afetadas pelo investimento em propaganda. As principais medidas identificadas foram: Q de Tobin (17); retorno total de acionista/retorno de ação (10); retorno anormal de curto prazo (6); market to book, receita de venda; retorno anormal de longo prazo e lucro/lucratividade (4). As demais medidas relacionam-se com satisfação dos consumidores, vendas, market-share, novos produtos, retorno dos ativos, fluxo de caixa, risco, imagem da empresa, crescimento da empresa e liquidez.

Nota-se uma utilização mais ampla de medidas de retornos de ações e Q de Tobin. O Q de Tobin é definido como "a razão entre o valor de mercado de uma empresa e o custo de reposição de seus ativos" (CHUNG e PRUITT, 1994, pg. 70; GERMANN, EBBES e GREWAL, 2015). Essa é também uma medida bem estabelecida para mensurar a proporção de valor intangível da empresa (SRIDHAR et al. 2016; WIES et al. 2019). Sridhar et al. (2016), apontam vantagens de utilizar Q de Tobin, são elas: incorpora informações sobre expectativa de ganhos futuros; não é afetada por convenções contábeis, mas ajustada a performance da indústria; compara várias organizações e indústrias, pois não é afetada pelos objetivos organizacionais; e não é vulnerável a interpretações da lei tributária.

O retorno anormal de ações pode ser uma proxy para expectativa dos investidores quanto a futuros desvios no fluxo de caixa em função de um evento (RAITHEL, TAYLOR e HOCK, 2016). Sirinivasan et al. (2009) entendem que as ações de marketing são ativos intangíveis que afetam a perspectiva dos investidores quanto ao fluxo de caixa futuro, refletindo o retorno de ações. A métrica utilizada pode afetar a elasticidade da propaganda no valor da firma (EDELING e FISCHER, 2016). Esses autores identificam que a elasticidade da propaganda tende a ser maior para medidas como $\mathrm{Q}$ de Tobin do que para retorno de ações. Os autores citam o estudo de Mizik e Jacobson (2009), para argumentar que o valor de Q de Tobin pode sofrer auto correlação e sugerem que a medida de retorno de ações é melhor variável dependente.

\subsection{ANÁLISE DAS CATEGORIAS TEMÁTICAS IDENTIFICADAS}

Foi possível identificar cinco categorias temáticas dentre os trabalhos avaliados. Identificou-se: (1) investimento em propaganda, (2) competição, (3) sinergia/canibalização, (4) 
propaganda enganosa e (5) gestão de crise. Uma análise mais detalhada acerca de cada um desses temas é feita a seguir.

Investimento em propaganda: O estudo de Edeling e Fischer (2016) trata-se de uma meta-análise do impacto do marketing no valor da firma. Além de identificar diferenças de resultados em função da métrica de valor da firma, os autores apontam que uma maior concentração de mercado leva a menor eficiência da propaganda no valor da firma. Os autores acreditam que o aumento do investimento de uma companhia na propaganda leva a uma reação de suas concorrentes, diminuindo os efeitos.

Competição: Considerando o ambiente competitivo destacam-se os achados de que o aumento do investimento em comunicação diminui o valor da firma de suas concorrentes de mesmo porte (JOSHI e HANSSENS, 2010). Ainda sobre o cenário competitivo, Joshi e Hanssens (2010) identificam que o mercado pune empresas em que o investimento em propaganda se desvia do nível ótimo. Eles consideram que, ao anunciarem, as empresas tornamse familiares aos acionistas, convertendo-se em opção mais atrativa de investimento. Já Fosfuri e Giarratana (2009), utilizam o registro de marca como proxy para nova publicidade em um mercado maduro e próximo a um duopólio. Os autores identificam que o registro de uma nova marca de uma empresa, aumenta a capitalização de mercado de sua concorrente. Os autores argumentam que em mercados maduros há maior lealdade e identidade dos consumidores com as marcas, assim todas as empresas se beneficiam da propaganda. Com base nesses estudos, se observa que os efeitos da propaganda no valor da firma das empresas concorrentes, apresentam um campo para futuros estudos que abordem quais situações a propaganda da concorrência afeta positivamente (negativamente), as métricas da empresa.

Sinergia/canibalização: Identificou-se o estudo de Sridhar et al. (2016). Nele os autores dividem as várias mídias em três grupos (nacional, regional e online), apresentando as diferenças quanto quantidade e qualidade do alcance, bem como a mensagem elaborada por cada uma. Os autores constatam que o valor da firma é impactado individualmente por cada ferramenta, contudo o aumento do investimento em uma ferramenta aumenta sua eficiência, contudo enfraquece a performance das demais ferramentas. Os autores acreditam que os distintos objetivos dessas mídias acabam por confundir o consumidor através de mensagens conflitantes. Ma e Du (2018) identificam que existe um nível ótimo da relação dos investimentos em propaganda digital com as mídias tradicionais no impacto do valor da firma. Ma e Du (2018) recomendam que as empresas devem investir concomitantemente em mídias digitais e tradicionais para maximizar o impacto da publicidade no valor da firma. 
Propaganda enganosa: A partir da metodologia de estudo de evento, Jeong e Yoo (2011) identificam que empresas que se envolvem em propaganda enganosa e são punidas pela Federal Trade Commission (FTC) experimentam um retorno anormal negativo de ações. Contudo, empresas com maior investimento em propaganda foram menos afetadas nessa relação - o investimento em comunicação apresenta-se como variável moderadora.

Gestão de crises: Uma possível explicação a esse efeito moderador apresentado anteriormente, pode ser observada na pesquisa classificada aqui como "gestão de crises" de Liu, Shankar e Yun (2017), estudo no qual se constatou que o volume de propaganda reduz os efeitos negativos de longo prazo do volume de recall e retorno anormal. Os autores argumentam que a ênfase em investimentos em publicidade pode demonstrar comprometimento em aumentar o valor da marca. Acredita-se que apesar de enviar um sinal negativo ao mercado ao se envolver em crise operacional ou de imagem, as empresas que investem mais nessa mídia demonstram intenção de aumentar o brand eqüity, diminuindo a percepção de perdas dos acionistas. Observa-se, assim, o papel moderador da propaganda como forma de reduzir efeitos negativos de tais tipos de crises.

\subsection{ANÁLISE DOS EFEITOS DE INVESTIMENTOS EM PROPAGANDA NA PERFORMANCE DA FIRMA}

Conforme supracitado, os resultados acerca dos efeitos de investimentos em propaganda na performance das empresas foram analisados de acordo com a métrica de performance da firma utilizada. Nas subseções a seguir, as análises são apresentadas.

\subsubsection{Propaganda e o $Q$ de Tobin}

Foram identificados 17 estudos nos quais o valor da firma foi mensurado com base no Q de Tobin. A maior parte dos estudos apontam para relação positiva entre o investimento em comunicação e essa métrica de valor da firma (12 artigos). Em alguns casos, foram identificados efeitos duplos (positivo e positivo/negativo) em função de análise conjunta e mitigada dos dados ou forma de mensuração. É possível observar a eficácia da propaganda no valor da firma, considerando o Q de Tobin como medida.

Malshe e Agarwal (2015) exploram o papel da alavancagem na satisfação do consumidor. Os autores identificam que a relação negativa entre alavancagem e satisfação é mediada pelos investimentos em propaganda. Os autores pontuam que o investimento em 
propaganda gera impactos positivos em diversas variáveis que antecedem a satisfação (por exemplo, qualidade percebida). Na continuação da análise, os autores observam que o valor da firma (medido pelo log de Q de Tobin) não é afetado pela propaganda (relação positiva, porém sem significância estatística). Da mesma forma Merino, Srinivasan e Srivastava (2006), por sua vez, identificam que o investimento em propaganda apresenta efeito positivo no Q de Tobin, contudo a relação é negativa quando considera-se a variabilidade do valor intangível (log de Q de Tobin). Identifica-se, assim, que a forma de mensurar e analisar a medida de performance pode influenciar o resultado.

Outro fator que pode afetar a relação entre comunicação e o valor da firma, é a estratégia da empresa (McALISTER et al. 2016). Os autores acreditam que as empresas que se utilizam da estratégia de diferenciação se beneficiam mais desse investimento, pois as comunicações utilizadas buscam criar algo diferenciado para o consumidor, e isso torna o cliente menos sensível às variações de preço e mais responsivo aos chamados da empresa. A estratégia tornase um fator de controle que pode afetar essa relação. Peng et al. (2018), identificam uma relação positiva, porém sem significância entre propaganda e o valor da firma, medido por meio do Q de Tobin. Contudo, os autores consideram o impacto do tamanho da empresa, medido a partir do total de ativos, e constatam a relação apenas em empresas maiores (vs. menores). Esses dois estudos atestam que algumas variáveis relacionadas com características das empresas podem afetar as relações propostas, devendo haver um controle por parte dos pesquisadores.

É importante ressaltar que, em sua maioria, os estudos sobre os efeitos do investimento em propaganda no valor da firma ocorrem com empresas e bases de dados nos Estados Unidos. Kundu, Kulkarni e Murthy (2010), porém, analisaram empresas do mercado indiano. Os autores identificam relação positiva da propaganda no valor da firma ( $Q$ de Tobin) foi positiva nos dois setores estudados (financeiro e de bens duráveis). Os autores contribuem ao indicar a necessidade de avaliar setores da indústria separadamente.

Por fim, destaca-se o estudo de Martin et al. (2018), que também constataram que a propaganda não apresenta relação significativa com o $\mathrm{Q}$ de Tobin. Adicionalmente, os autores identificaram que a propaganda gera efeitos negativos no risco sistemático, relacionado ao mercado no qual a empresa atua, e efeitos positivos no risco idiossincrático, relacionado ao risco específico de uma empresa. Embora a análise do risco não seja o foco da presente pesquisa, se faz relevante ressaltar que poucos estudos consideram o efeito da comunicação no risco do mercado e do empresa. 


\subsubsection{Propaganda e o retorno de ações}

Ao tratar sobre medidas de retorno de ações (sejam retornos anormais ou total), observou-se 20 ocorrências dessas medidas. Em cinco vezes falhou-se em achar a relação positiva entre o investimento em propaganda e essas medidas, sendo dois para retornos anormais de curto prazo, dois para retorno total de acionistas/retorno de ação e uma utilizandose retorno anormal de longo prazo.

O retorno de ações é impulsionado por notícias no fluxo de caixa esperado ou nas taxas de retorno (VUOLTEENAHO, 2002). Por sua vez, o retorno anormal são àqueles que estão acima dos retornos esperados (COLICEV et al. 2018).

Miyazaki e Morgan (2001), testam o efeito do anúncio da propaganda nos jogos olímpicos sobre o retorno de ações por meio de um estudo de eventos. Os autores indicam que, apesar da crítica a esse tipo de estratégia, seus resultados falham em achar retornos negativos significantes, e, em uma janela de tempo analisada, encontram um resultado positivo, sugerindo que investir em grandes eventos, como os jogos olímpicos, aparenta ser uma estratégia justificável. Entretanto, deve-se notar que os resultados, em geral, apontam para efeito neutro.

Outro estudo demonstra que o impacto da propaganda na final do campeonato de futebol americano (Super Bowl) sobre o retorno de ações só é positivo por meio do aumento do brand equity baseado no consumidor (RAITHEL, TAYLOR e HOCK, 2016). Os autores afirmam que estudos anteriores sobre a comunicação no Super Bowl apresentam resultados mistos e argumentam a necessidade de se testar variáveis moderadoras/mediadores, como o brand equity baseado no consumidor. Dessa forma, é possível que a falha, de forma geral, em achar resultados positivos significativos no patrocínio das olimpíadas (MIYAZAKI e MORGAN, 2001) se associe a não consideração de variáveis moderadoras e mediadoras (RAITHEL, TAYLOR e HOCK, 2016).

Ao considerar o retorno total dos acionistas, Tuli, Mukherjee e Dekimpe (2012) apontam que o investimento em propaganda no setor de varejo só afetará positivamente o valor da firma na presença de ganhos inesperados ${ }^{11}$. Esse resultado vai de encontro ao estudo de Ho, Keh e Ong (2005), que observam que empresas não-manufatureiras se beneficiam mais de investimento em propaganda, frente empresas de manufatura. Os autores argumentam que empresas não-manufatureiras podem não utilizar $P \& D$ e assim focar na propaganda. Nota-se,

\footnotetext{
${ }^{11}$ Reflete a performance em um período e a capacidade de gerar receitas (TULI, MUKHERJEE e DEKIMPE 2012).
} 
com base em ambos estudos que, apesar de aparentemente empresas não-manufatureiras se beneficiarem mais dos investimentos em propaganda, resultados contrários também são observados. Identifica-se, assim, que as relações podem ser afetadas pelos distintos setores estudados.

Por fim, em um dos poucos estudos avaliados que foram realizados fora do mercado norte americano, Han e Manry $\left(2004^{12}\right)$ analisaram empresas da Coréia do Sul. Os autores apontaram resultados negativos do investimento em propaganda no preço de ações. Resultados como esse, portanto, evidenciam uma potencial influência da cultura local no efeito em questão.

\subsubsection{Propaganda e Market-to-book, lucro, receita e variáveis culturais}

Em geral, os resultados apontam para a eficácia da propaganda em medidas relevantes, como lucro e receita, porém essas medidas não são necessariamente relacionadas com o mercado financeiro (KATSIKEAS et al. 2016). Como exceção, verificou-se o estudo de Bayus, Erickson e Jacobson (2003), que não encontraram efeitos positivos da propaganda de um novo produto na lucratividade da empresa. Espera-se que o investimento em propaganda afete positivamente essas medidas, pois conforme apresentam Srinivasan et al. (2009), diversos estudos demonstram o resultado positivo nessas medidas, aparentando um consenso da eficácia da comunicação.

Com relação a medida market-to-book, nota-se uma menor utilização (quatro estudos). O market-to-book é calculado por meio da razão entre o valor de mercado de ações de uma empresa e o valor contábil de seus ativos (SRINIVASAN et al. 2013; TELLIS, PRABHU e CHANDY, 2009). Entre os resultados encontrados, cabe destacar os achados de Srinivasan et al. (2013), os quais apontam que a maior propaganda intensifica a relação entre abertura (fechamento) de loja e menor (maior) valor da firma. Os autores apontam que o fechamento de lojas indica aos investidores que a cadeia irá se focar no atual núcleo de consumidores. Os demais estudos apresentam efeito positivo dos investimentos em propaganda no market-tobook.

A principal contribuição da análise de resultados realizada foi demonstrar que a relação entre investimento em propaganda e o valor da firma pode apresentar várias nuances, não existindo uma relação generalizada a todo tipo de companhia. Reitera-se, assim, o argumento

\footnotetext{
12 Artigos utilizados para embasar resultados descobertos, porém não selecionados na revisão sistemática em função dos critérios utilizados.
} 
de Assaf et al. (2017) que afirmam que essa relação (investimento em propaganda e valor da firma) não é direta. Há de se considerar ainda que as empresas possuem recursos limitados e várias áreas a receberem investimentos, devendo assim buscar um nível ótimo de alocação de recursos de forma a obter máximo valor da firma.

\section{AGENDA DE PESQUISA}

Ainda que diversos temas já tenham sido abordados e alguns posicionamentos sedimentados na área, a revisão feita foi capaz de identificar alguns tópicos nos quais fica evidenciada a necessidade de estudos futuros. Nesta sessão, é apresentada uma agenda de pesquisa para nortear quais direcionamentos os pesquisadores da área podem aproveitar, afim de diminuir as lacunas existentes na área.

Variáveis moderadoras. Nota-se um chamado na literatura para estudos que considerem variáveis moderadoras que afetem o caminho entre o investimento em propaganda e o valor da firma (e.g. Assaf et al. 2017; Edeling e Fisher, 2016). Esse chamado se justifica no presente estudo ao demonstrar que elementos como a estratégia e o setor podem afetar essa relação. Shah e Akbar (2008) atentam para novos estudos em distintos setores como forma a observar em quais os resultados são maiores. Ainda, os estudos não consideram a presença ou ausência de endossantes que podem intensificar (ou diminuir) a relação. Também não se observou nos estudos uma análise do histórico da empresa (se já se envolveu em propagandas enganosas no passado, por exemplo). Há de se considerar também o público alvo (jovens, idosos, homens, mulheres, etc.) para entender se o investimento em comunicação aos diferentes públicos apresenta efeitos diversos no valor da firma.

Outra variável que deve ser melhor verificada é a cultura. Em um primeiro momento nota-se uma escassez de estudos realizados no Brasil, por exemplo. A partir da revisão, identificou-se apenas o estudo de Fortunato et al. (2010), que realizam a análise com base em empresas norte americanas. Com isso, gestores e investidores brasileiros baseiam suas decisões a partir de pesquisas com empresas de outros países. Contudo, os resultados demonstram uma potencial diferença cultural. Este estudo encoraja pesquisadores brasileiros a realizarem mais análises com empresas nacionais. Adicionalmente, essa concentração de pesquisas sobre o mercado norte americano proporciona a oportunidade de, além do Brasil, considerar também outros países com o objetivo de comparar os efeitos do investimento em propaganda no valor 
da firma entre diferentes mercados nacionais. Isso proporcionaria uma maior generalização acerca do fenômeno.

Canibalização/sinergia. Para a análise de canibalização/sinergia, seria importante que novas pesquisas buscassem ampliar as ferramentas do mix de comunicação para além daquelas identificadas na revisão, possibilitando a identificação de um nível ótimo de investimento. Algumas ferramentas, como patrocínio por exemplo, foram identificadas ao decorrer da análise. Futuras pesquisas deveriam considerar várias fontes de publicidade da empresa de forma conjunta para testar seus impactos no valor da firma.

Áreas adicionais de investimento. Se faz relevante considerar outras áreas de investimento nas empresas, além de comunicação, buscando identificar melhor a performance a partir de múltiplas ferramentas. Ou seja, os gestores devem alocar seus recursos em distintas áreas na gestão, como gestão de estoques e pesquisa e desenvolvimento (por exemplo, SRIDHAR, NARAYANAN e SRINIVASAN, 2014). Os pesquisadores devem considerar um maior volume de variáveis (que não apenas investimento em propaganda), buscando estimar níveis ótimos de investimento para a maximização do valor da firma.

Efeito sobre competidores. Os estudos acerca da categoria temática denominada competição evidenciam que algumas pesquisas já comprovaram efeitos relevantes da propaganda sobre as empresas concorrentes. Estender os achados desses estudos é um caminho relevante para a área de marketing, principalmente na medida em que há um aumento da competitividade em muitos mercados. Dessa forma, o invés de analisar o efeito da propaganda sobre o valor de concorrentes em mercados altamente concentrados, conforme já realizado, há a oportunidade de avaliar tal efeito em mercado altamente competitivos.

Eventos. A análise evidenciou que algumas pesquisas demonstram os impactos de anúncios em grandes eventos como os jogos olímpicos (MIYAZAKI e MORGAN, 2001). Os estudos analisados também concentram seus resultados em economias desenvolvidas (Estados Unidos principalmente). Diante disso, algumas oportunidades de pesquisas são: replicar esses resultados em economias em desenvolvimento, como o Brasil por exemplo; e verificar quais eventos geraram mais retorno para empresas de países emergentes, ou seja, eventos esportivos, societais, entre outros.

\section{CONSIDERAÇÕES FINAIS}


Neste estudo, realizou-se uma revisão sistemática acerca da relação entre investimento em propaganda e comunicação e o valor da firma. A partir de uma base final de 43 artigos produzidos de 1997 a 2019, foi possível observar que, em geral, medidas que se associam a métricas relevantes, contudo não associadas diretamente ao mercado financeiro, apontam resultados positivos ao efeito da propaganda. Para medidas de mercado financeiro os resultados apontam em sua maioria para efeitos positivos, porém demonstrou-se que relações insignificantes e negativas podem ser encontradas, indicando a necessidade de maiores pesquisas para compreender melhor tal relação.

Os resultados demonstram que existem variáveis que devem ser conhecidas e controladas, pois elas podem exercer influência na relação entre propaganda e valor da firma (a partir de moderação ou mediação). Uma maior generalização desses estudos seria de grande valia aos executivos, ao demonstrar em quais setores, condições, culturas, etc. a propaganda gera melhores resultados ao valor da firma. Essas questões estão expostas na agenda de pesquisa proposta.

Este estudo traz contribuições importantes à academia. Inicialmente, deu-se continuidade ao estudo de Shah e Akbar (2008), que possuía como limitação a análise de artigos até 2004. Este artigo expande esta análise, avaliando publicações entre 1997 e 2019. Além disso, foi possível compilar e apresentar motivos pelos quais a relação entre propaganda e o valor da firma não podem ser generalizados. Este artigo também contribui à academia ao propor uma agenda para pesquisas futuras, apontando os caminhos de pesquisa mais relevantes para a redução de lacunas do conhecimento.

Além de sua contribuição acadêmica, este artigo também possui implicações gerenciais. Esta pesquisa apresentou situações onde valor da firma não é afetado pelos investimentos em propaganda e pode servir de guia à gestores quanto ao investimento ou não nessa ferramenta.

O presente estudo buscou seguir uma metodologia de revisão sistemática, contudo algumas limitações, inclusive inerentes à metodologia, são observadas. Uma primeira limitação encontra-se nas palavras usadas na busca (advertsing e firm value), novos estudos podem considerar outras como firm performance; metrics; shareholder value; além dos nomes das métricas (Q de Tobin, por exemplo). Outra limitação ocorre no foco do estudo ao atentar-se aos investimentos em comunicação, não levando em consideração outros investimentos.

Novos estudos podem mapear outras variáveis como investimento em pesquisa e desenvolvimento, responsabilidade social, entre outros. Ressalva-se que, na presente análise, alguns artigos debatiam concomitantemente esses tópicos, porém os resultados não foram 
reportados em função do escopo. Sugere-se ainda que futuras pesquisas busquem preencher as lacunas apresentadas na agenda de pesquisa proposta.

\section{REFERÊNCIAS}

AMBLER, Tim; KOKKINAKI, Flora; PUNTONI, Stefano. Assessing marketing performance: reasons for metrics selection. Journal of Marketing Management, v. 20, n. 3-4, p. 475-498, 2004.

ASSAF, A. George et al. Advertising spending, firm performance, and the moderating impact of CSR. Tourism Economics, v. 23, n. 7, p. 1484-1495, 2017.

BAYUS, Barry L.; ERICKSON, Gary; JACOBSON, Robert. The financial rewards of new product introductions in the personal computer industry. Management Science, v. 49, n. 2, p. 197-210, 2003.

BENDLE, Neil et al. Marketing metrics: The manager's guide to measuring marketing performance. FT Press, 2015.

BRINCKMANN, Jan; GRICHNIK, Dietmar; KAPSA, Diana. Should entrepreneurs plan or just storm the castle? A meta-analysis on contextual factors impacting the business planningperformance relationship in small firms. Journal of business Venturing, v. 25, n. 1, p. 24-40, 2010.

BROILO, Patricia Liebesny; ESPARTEL, Lélis Balestrin. Confusão do Consumidor no Cenário Multimídia e Multicanal: Definição do Construto e Agenda de Pesquisa. REMark, v. 15, n. 1, p. 42, 2016.

CHANG, Woojung; TAYLOR, Steven A. The effectiveness of customer participation in new product development: A meta-analysis. Journal of Marketing, v. 80, n. 1, p. 47-64, 2016.

CHEONG, Yunjae; DE GREGORIO, Federico; KIM, Kihan. Advertising spending efficiency among top US advertisers from 1985 to 2012: Overspending or smart managing? Journal of Advertising, v. 43, n. 4, p. 344-358, 2014.

CHRISTODOULIDES, George; DE CHERNATONY, Leslie. Consumer-based brand equity conceptualization and measurement: A literature review. International Journal of Research in Marketing, v. 52, n. 1, p. 43-66, 2010.

CHUNG, Kee H.; PRUITT, Stephen W. A simple approximation of Tobin's q. Financial management, p. 70-74, 1994. 
COLICEV, Anatoli et al. Improving consumer mindset metrics and shareholder value through social media: The different roles of owned and earned media. Journal of Marketing, v. 82, n. 1, p. 37-56, 2018.

DATTA, H.; AILAWADI, K. L.; VAN HEERDE, H. J. How Well Does Consumer-Based Brand Equity Align with Sales-Based Brand Equity and Marketing-Mix Response? Journal of Marketing, v. 81, n. 3, p. 1-20, 2017.

EDELING, Alexander; FISCHER, Marc. Marketing's impact on firm value: Generalizations from a meta-analysis. Journal of Marketing Research, v. 53, n. 4, p. 515-534, 2016.

FETSCHERIN, Marc; HEINRICH, Daniel. Consumer brand relationships research: A bibliometric citation meta-analysis. Journal of Business Research, v. 68, n. 2, p. 380-390, 2015.

FORTUNATO, Graziela et al. Interacao dos dispendios de publicidade com ciclos economicos e o valor da empresa: analise empirica das empresas Norte-Americanas do setor de bens de consumo. Revista Brasileira de Finanças, v. 8, n. 3, 2010.

FOSFURI, Andrea; GIARRATANA, Marco S. Masters of war: Rivals' product innovation and new advertising in mature product markets. Management Science, v. 55, n. 2, p. 181-191, 2009.

FRENNEA, Carly; HAN, Kyuhong; MITTAL, Vikas. Value Appropriation and Firm Shareholder Value: Role of Advertising and Receivables Management. Journal of Marketing Research, v. 56, n. 2. p. 291-309, 2019.

GERMANN, Frank; EBBES, Peter, GREWAL, Rajdeep. The Chief Marketing Officer Matters! Journal of Marketing, v. 79, n. 3, p. 1-22, 2015.

GÖK, O.; HACIOGLU, G. The organizational roles of marketing and marketing managers. Marketing Intelligence \& Planning, v. 28, v. 3, p. 291-309, 2010.

HAN, Bong H.; MANRY, David. The value-relevance of R\&D and advertising expenditures: Evidence from Korea. The International Journal of Accounting, v. 39, n. 2, p. 155-173, 2004. HANSSENS, Dominique M.; RUST, Roland T.; SRIVASTAVA, Rajendra K. Marketing strategy and Wall Street: nailing down marketing's impact. Journal of Marketing, v. 73, n. 6, p. $115-118,2009$.

HO, Yew Kee; KEH, Hean Tat; ONG, Jin Mei. The effects of R\&D and advertising on firm value: an examination of manufacturing and nonmanufacturing firms. IEEE Transactions on Engineering Management, v. 52, n. 1, p. 3-14, 2005. 
HOMBURG, Christian et al. The loss of the marketing department's influence: is it really happening? And why worry?. Journal of the Academy of Marketing Science, v. 43, n. 1, p. $1-13,2015$.

HYMAN, Michael R.; MATHUR, Ike. Retrospective and prospective views on the marketing/finance interface. Journal of the Academy of Marketing Science, v. 33, n. 4, p. 390-400, 2005.

JEONG, Jaeseok; YUN YOO, Chan. Deceptive advertising and abnormal stock returns: An event study analysis. International Journal of Advertising, v. 30, n. 3, p. 509-535, 2011.

JOHNSON, Robert; SOENEN, Luc. Indicators of successful companies. European Management Journal, v. 21, n. 3, p. 364-369, 2003.

JOSHI, Amit; HANSSENS, Dominique M. The direct and indirect effects of advertising spending on firm value. Journal of Marketing, v. 74, n. 1, p. 20-33, 2010.

KATSIKEAS, Constantine S. et al. Assessing performance outcomes in marketing. Journal of Marketing, v. 80, n. 2, p. 1-20, 2016.

KIM, YongHee; KIM, MinChung; O'NEILL, John W. Advertising and firm risk: A study of the restaurant industry. Journal of Travel \& Tourism Marketing, v. 30, n. 5, p. 455-470, 2013.

KITCHEN, Philip J. "Guest editorial", European Journal of Marketing, v. 51 n. 3, p.394405, 2017.

KNOWLES, Jonathan; AMBLER, Tim. Orientation and Marketing Metrics. The SAGE Handbook of Marketing Theory, p. 379, 2009.

KUMAR, V. Evolution of marketing as a discipline: What has happened and what to look out for. Journal of Marketing, v. 79, n. 1, p. 1-9, 2015.

KUMAR, V. Integrating theory and practice in marketing. Journal of Markeing, v. 81, n. 2, p. 1-7, 2017.

KUNDU, Anindita; KULKARNI, Prashant; NK, Anantha Murthy. Advertising and firm value: mapping the relationship between advertising, profitability and business strategy in India. Changing Ideas in Strategy (eds: Arun Sinha), Narosa Publishing, 2010.

LIU, Yan; SHANKAR, Venkatesh; YUN, Wonjoo. Crisis management strategies and the longterm effects of product recalls on firm value. Journal of Marketing, v. 81, n. 5, p. 30-48, 2017. MA, Judy; DU, Brian. Digital Advertising And Company Value: Implications of Reallocating Advertising Expenditures. Journal of Advertising Research, v. 58, n. 3, p. 326-337, 2018. 
MALSHE, Ashwin; AGARWAL, Manoj K. From finance to marketing: the impact of financial leverage on customer satisfaction. Journal of Marketing, v. 79, n. 5, p. 21-38, 2015.

MARTIN, Kelly D. et al. Political Management, Research and Development, and Advertising Capital in the Pharmaceutical Industry: A Good Prognosis?. Journal of Marketing, v. 82, n. 3, p. 87-107, 2018.

MCALISTER, Leigh et al. Advertising effectiveness: the moderating effect of firm strategy. Journal of Marketing Research, v. 53, n. 2, p. 207-224, 2016.

MERINO, Maria; SRINIVASAN, R.; SRIVASTAVA, R. K. Advertising, R\&D, and variability of cash flow and intangible firm value. Unpublished manuscript, 2006.

MIZIK, Natalie; JACOBSON, Robert. Financial Markets Research in Marketing. In: Commentaries and Rejoinder to 'Marketing and Firm Value: Metrics, Methods, Findings and Future Directions', Journal of Marketing Research, v. 46, n. 3, p. 320-24, 2009.

MIYAZAKI, Anthony D.; MORGAN, Angela G. Assessing the Market Value of Sponsoring: Corporate Olympic Sponsorships. Journal of Advertising Research, v. 41, n. 1, p. 9-15, 2001. MOORMAN, Christine et al. JM as a Marketplace of Ideas. Journal of Marketing, v. 83, n. 1, p. 1-7, 2019.

MORGAN, Neil A. Marketing and business performance. Journal of the Academy of Marketing Science, v. 40, n. 1, p. 102-119, 2012.

OLIVEIRA, Marta O. R.; SILVEIRA, Cleo Schmitt; LUCE, Fernando Bins. Brand equity estimation model. Journal of Business Research, v. 68, n. 12, p. 2560-2568, 2015.

OLIVEIRA, Marta O. R. et al. Interface entre marketing e finanças: um levantamento sobre indicadores de desempenho e aproximação entre as essas áreas. Anais do Encontro Nacional da Associação Nacional de Pós-Graduação e Pesquisa em Administração, 2010.

OSINGA, Ernst C. et al. Why do firms invest in consumer advertising with limited sales response? A shareholder perspective. Journal of Marketing, v. 75, n. 1, p. 109-124, 2011.

PALMATIER, Robert W.; HOUSTON, Mark B.; HULLAND, John. Review articles: purpose, process, and structure. Journal of the Academy of Marketing Science, v. 46, n. 1, p. 1-5, 2018 PENG, Chi- Lu et al. Synergy between R\&D and advertising on shareholder value: Does firm size matter? Canadian Journal of Administrative Sciences/Revue Canadienne des Sciences de l'Administration, v. 35, n. 1, p. 47-64, 2018.

PETERSON, Robert A.; JEONG, Jaeseok. Exploring the impact of advertising and R\&D expenditures on corporate brand value and firm-level financial performance. Journal of the Academy of Marketing Science, v. 38, n. 6, p. 677-690, 2010. 
RAITHEL, Sascha; TAYLOR, Charles R.; HOCK, Stefan J. Are Super Bowl ads a super waste of money? Examining the intermediary roles of customer-based brand equity and customer equity effects. Journal of Business Research, v. 69, n. 9, p. 3788-3794, 2016.

REIBSTEIN, David J.; DAY, George; WIND, Jerry. Guest editorial: is marketing academia losing its way?. Journal of Marketing, v. 73, n. 4, p. 1-3, 2009.

RUST, Roland T. et al. Measuring marketing productivity: Current knowledge and future directions. Journal of marketing, v. 68, n. 4, p. 76-89, 2004.

SHAH, Syed Zulfiqar Ali; AKBAR, Saeed. Value relevance of advertising expenditure: A review of the literature. International Journal of Management Reviews, v. 10, n. 4, p. 301$325,2008$.

SHETH, J. N.; SISODIA, R. S. Feeling the heat - part 1. Marketing Management, v. 4, n. 2, p. 9-23, 1995a.

SHETH, J. N.; SISODIA, R. S. Feeling the heat - part 2. Marketing Management, v. 4, n. 3, p. 19-33, 1995b.

SHIMP, Terence A.; ANDREWS, J. Craig. Advertising promotion and other aspects of integrated marketing communications. Cengage Learning, 2013.

SRIDHAR, Shrihari; NARAYANAN, Sriram; SRINIVASAN, Raji. Dynamic relationships among $R \& D$, advertising, inventory and firm performance. Journal of the Academy of Marketing Science, v. 42, n. 3, p. 277-290, 2014.

SRIDHAR, Shrihari; et al. Relating online, regional, and national advertising to firm value. Journal of Marketing, v. 80, n. 4, p. 39-55, 2016.

SRINIVASAN, Raji et al. Effects of opening and closing stores on chain retailer performance. Journal of Retailing, v. 89, n. 2, p. 126-139, 2013.

SRINIVASAN, Shuba; HANSSENS, Dominique M. Marketing and firm value: Metrics, methods, findings, and future directions. Journal of Marketing Research, v. 46, n. 3, p. 293$312,2009$.

SRINIVASAN, Shuba et al. Product innovations, advertising, and stock returns. Journal of Marketing, v. 73, n. 1, p. 24-43, 2009.

SRIVASTAVA, R. K.; SHERVANI, T. A.; FAHEY, L. Market-based assets and shareholder value: A framework for analysis. Journal of Marketing, v. 62, n. 1, p. 2-18, 1998.

STEWART, David W. Marketing accountability: Linking marketing actions to financial results. Journal of Business Research, v. 62, n. 6, p. 636-643, 2009. 
TAYLOR, Charles, Editorial, International Journal of Advertising: The Review of Marketing Communications, v. 29, n. 2, p. 161-164, 2010.

TELLIS, Gerard J.; PRABHU, Jaideep C.; CHANDY, Rajesh K. Radical innovation across nations: The preeminence of corporate culture. Journal of Marketing, v. 73, n. 1, p. 3-23, 2009.

TULI, Kapil R.; MUKHERJEE, Anirban; DEKIMPE, Marnik G. On the value relevance of retailer advertising spending and same-store sales growth. Journal of Retailing, v. 88, n. 4, p. 447-461, 2012.

VUOLTEENAHO, Tuomo. What drives firm- level stock returns?. The Journal of Finance, v. 57, n. 1, p. 233-264, 2002.

WIES, Simone et al. Can Advertising Investments Counter the Negative Impact of Shareholder Complaints on Firm Value?. Journal of Marketing, v. 83, n. 4, p. 58-80, 2019.

WOLFSWINKEL, Joost F.; FURTMUELLER, Elfi; WILDEROM, Celeste PM. Using grounded theory as a method for rigorously reviewing literature. European Journal of Information Systems, v. 22, n. 1, p. 45-55, 2013. 